Mens

revue d'histoire intellectuelle de l'Amérique française

\title{
Les Canadiens français, l'histoire et la démocratie : l'interprétation de Pierre Elliott Trudeau
}

\section{Mélanie Ouellette}

Volume 1, numéro 1, automne 2000

URI : https://id.erudit.org/iderudit/1024438ar

DOI : https://doi.org/10.7202/1024438ar

Aller au sommaire du numéro

Éditeur(s)

Centre de recherche en civilisation canadienne-française

ISSN

1492-8647 (imprimé)

1927-9299 (numérique)

Découvrir la revue

Citer cet article

Ouellette, M. (2000). Les Canadiens français, l'histoire et la démocratie :

l'interprétation de Pierre Elliott Trudeau. Mens, 1(1), 37-50.

https://doi.org/10.7202/1024438ar

\section{Résumé de l'article}

Pierre Trudeau s'emploie à dresser, au cours de la décennie 1950, un portrait fort critique de l'état de la démocratie au Canada français. À son avis, le peuple canadien-français a, de tout temps, fait de la démocratie une tactique, plutôt qu'un instrument privilégié de développement social. D’où, pour l'auteur, une tendance marquée chez les francophones à l'immoralisme social et politique, à la corruption et aux manigances électorales. Parce qu'ils ont en quelque sorte hérité du régime démocratique, qu'ils n'ont pas eu à combattre pour celui-ci, les Canadiens français n'ont jamais pu véritablement l'assimiler. Ils ont plutôt perverti le système, aidés en ce sens tantôt par le conquérant anglais, bien décidé à profiter de cette méconnaissance démontrée par la minorité vaincue, tantôt par l'Église catholique, pour qui la démocratie allait de pair avec socialisme, révolution, athéisme... Pour Trudeau, le temps est venu, à la fin des années 1950, de jeter aux orties ces anciens préjugés, pour enfin accueillir un régime véritablement démocratique, libéré de l’influence néfaste de ses détracteurs. 


\title{
LES CANADIENS FRANÇAIS, L'HISTOIRE ET LA DÉMOCRATIE : L'INTERPRÉTATION DE PIERRE ELLIOTT TRUDEAU
}

\author{
MÉLANIE OUELLETTE \\ Candidate à la maîtrise \\ Département d'histoire \\ Université de Montréal
}

\section{RÉSUMÉ}

Pierre Trudeau s'emploie à dresser, au cours de la décennie 1950, un portrait fort critique de l'état de la démocratie au Canada français. À son avis, le peuple canadienfrançais a, de tout temps, fait de la démocratie une tactique, plutôt qu'un instrument privilégié de développement social. D'où, pour l'auteur, une tendance marquée chez les francophones à l'immoralisme social et politique, à la corruption et aux manigances électorales. Parce qu'ils ont en quelque sorte bérité du régime démocratique, qu'ils n'ont pas eu à combattre pour celui-ci, les Canadiens français n'ont jamais pu véritablement l'assimiler. Ils ont plutôt perverti le système, aidés en ce sens tantôt par le conquérant anglais, bien décidé à profiter de cette méconnaissance démontrée par la minorité vaincue, tantôt par l'Église catholique, pour qui la démocratie allait de pair avec socialisme, révolution, athéisme... Pour Trudeau, le temps est venu, à la fin des années 1950 , de jeter aux orties ces anciens préjugés, pour enfin accueillir un régime véritablement démocratique, libéré de l'influence néfaste de ses détracteurs.

\section{ABSTRACT}

During the 1950', Pierre Trudeau was bighly critical of the state of democracy in French Canada. In his mind, French Canadians had always viewed democracy in a strategic and opportunistic fashion instead of embracing it as a tool for social development. Concretely, this opportunism expressed itself through electoral corruption and a distinct lack of social and political morality. French Canadians were unable to properly appreciate the merits of democracy because they had never been obliged to fight for it. Instead, they had, in a way, simply inherited it. Thus, they perverted a system they neither understood nor appreciated. In this endeavour, they were initially encouraged by the English conqueror, who exploited French Canada's lack of democratic experience to ensure bis dominance, and by the Roman Catholic Church, which equated democracy with socialism, atheism and revolution. By the end of the fifties, it 
became clear to Trudeau that the time had come to reject these antiquated prejudices. French Canadians would have to move beyond their illiberal tendencies and embrace true democracy.

Citoyen de ce pays, et avec ces principes, j’aurais été Canadien français d'adoption, si je ne l'avais déjà été de naissance. Et le Canada français eûtil manqué de gens pour lui prêcher la fierté collective, $j$ 'aurais sans doute été à la pointe du combat! Mais, grands dieux! nous n'avons eu que cela, des prédicateurs de fierté et des prophètes de mission providentielle. Nous abondions en vastes synthèses, nous construisions des superstructures élaborées, nous raffolions de réformes constitutionnelles ou juridiques, dont le plus clair du mérite était de n'avoir aucune prise sur le réel ${ }^{1}$.

Pierre Trudeau écrivait ces lignes en 1967, deux ans après son entrée en politique active. S'y trouvait résumée une bonne partie des critiques énoncées quelques années auparavant dans les pages de Cité libre et de Vrai: la dénonciation des «élucubrations ${ }^{2}$ » historiques des élites intellectuelles du Canada français, l'irréalisme politique et la nonchalance démocratique de ses dirigeants, l'absence d'adéquation des théories élaborées par ses penseurs au vécu quotidien de ses citoyens. Au cours de la décennie précédente, Trudeau s'était en effet employé à remettre en question les postulats historiques traditionnels, à démolir les unes à la suite des autres les thèses traditionalistes, qui sans cesse renvoyaient à la nécessité d'assurer la survivance et qui, le plus souvent, mettaient en opposition les spécificités canadiennes-françaises et les réalités d'une société participant désormais du umonde moderne». L'intellectuel se réclamait d'un progressisme moral, matériel et idéologique, et exhortait ses compatriotes à le suivre dans cette voie.

Pour Gérard Pelletier, son collaborateur le plus proche, le Pierre Trudeau d'alors représentait le parfait prototype de «l'homme nouveau» qui émergeait peu à peu. Il était de cette

[...] nouvelle espèce de Québecois [sic], plus préoccupée de liberté et de démocratie que de nationalisme, davantage soucieuse de moderniser nos institutions canadiennes-françaises que de les défendre, et préoccupée de ne pas subir les transformations économiques profondes qui bouleversaient le Québec, mais au contraire de les comprendre et de les diriger ${ }^{3}$.

N'était-ce pas en effet cet intellectuel qui, dès son premier article pour Cité libre, invitait ses lecteurs à faire «table rase ${ }^{4}$ » des vieilles idéologies, le 
temps étant venu selon lui «de jeter aux orties les mille préjugés dont le passé encombre le présent, et de bâtir pour l'homme nouveau"» ? Aux yeux de Pierre Trudeau, le moment était arrivé pour les Canadiens français de «déconstruire» une histoire jusque-là mythifiée.

Le présent article entend se pencher spécifiquement sur cette «lutte» à l'exaltation du passé que va mener Pierre Trudeau à la fin des années 1950. Une analyse des postulats fondamentaux de la vision historique de celui-ci sera ainsi proposée : c'est cette vision, singulière à bien des égards comme nous le verrons, qui sera à la base des critiques souvent virulentes adressées par le directeur de Cité libre à ses opposants. Car le théoricien politique cédera souvent la place, en ce domaine, à l'intellectuel polémiste : les propos trudeauistes sur l'origine des régimes démocratiques, neutres de prime abord, serviront souvent, nous le constaterons, de prémisses à de violentes attaques contre l'idéologie traditionaliste en apparence encore dominante à l'époque. D'où, pour une bonne part, la virulence des débats intellectuels associés à la fin du régime duplessiste, débats dont Pierre Trudeau sera l'un des principaux protagonistes.

Lorsqu'il présente, le 2 octobre 1954 (à l'occasion de la Première Conférence annuelle de l'Institut canadien des affaires publiques), une communication portant sur les "Obstacles à la démocratie $e^{6}$ " au Canada français, Pierre Trudeau ne fait que poursuivre un cheminement intellectuel déjà amorcé dans les pages de Cité libre et qu'il verra à étoffer au cours de la seconde moitié de la décennie 1950. Il y soumet en effet, pour la première fois à un large public ${ }^{8}$, l'une des thèses principales de sa pensée politique : les Canadiens français n'ont jamais assimilé autre chose qu'une démocratie «de façade», tronquée de ses postulats fondamentaux, et la cause première de cet état de fait est à rechercher dans leur histoire particulière.

Ce qu'expose Trudeau en 1954 (et ce sur quoi il ne cessera de bâtir par la suite), c'est le long processus, inéluctable, au terme duquel s'établit, dans une société donnée, une démocratie complète, viable et parfaitement intégrée par les citoyens. Ainsi, pour ce dernier, tout régime démocratique se développe en deux temps: pour être pleinement «réalisée» ou «établie», la démocratie doit au préalable avoir fait l'objet d'un "combat'». Le développement historique des sociétés démocratiques nous enseigne, rappelle Trudeau, que cette dernière forme 
de gouvernement n'est pas innée chez l'homme: la démocratie s'acquiert, souvent au prix de longues et difficiles luttes. Les régimes despotiques, absolutistes ou oligarchiques, parce qu'ils portent atteinte à la moralité sociale d'un peuple en définissant le bien et le mal en fonction de la volonté d'une seule personne (ou d'un groupe restreint de personnes), plutôt qu'en regard de celle de la majorité des citoyens, minent l'inviolabilité de l'être humain. Ils se substituent en effet à la conscience individuelle en déterminant à l'avance les rapports sociaux et la destinée collective, ils enlèvent à leurs citoyens toute liberté de choix (et par conséquent toute possibilité de poser un acte moral) en décidant unilatéralement guerres, paix, vie ou mort. Peu à peu toutefois, et parce que leur intégrité morale et physique se voit menacée, les gouvernés en viennent à s'interroger sur les fondements du pouvoir en place. Et, parfois confusément dans un premier temps puis de plus en plus clairement, ils en arrivent à poser le principe de l'égalité de tous les hommes : s'amorce dès lors la contestation d'une autorité omnipotente qui, sans qu'elle en soit de plein droit justifiée, est susceptible de gouverner les citoyens contre leur grê $e^{10}$. C'est le stade de la démocratie «combattante» :

Il en suit que la fonction de l'autorité ne doit pas s'exercer à proprement parler par le commandement, mais par la persuasion; et dès lors que les citoyens, dans leur ensemble, n'ont plus conscience d'être justement gouvemés, ils ont le droit de changer le gouvemement, pacifiquement s'ils en ont les moyens, et par la force dans le cas contraire ${ }^{11}$.

Une fois les obstacles extérieurs abattus, le nouveau régime doit voir à s'accomplir, à s'assurer l'adhésion totale de tous ses citoyens. Ces derniers expérimentent alors ce que Pierre Trudeau appelle le «second stade du développement de la démocratie ${ }^{12}$ ) : celui pendant lequel la récente forme de gouvernement s'《établio, se «réalise». De simple instrument mis au service de la raison d'État, l'homme se transforme et devient le moteur de son propre accomplissement. Il apparait désormais "comme une fin en lui-même ${ }^{13}$ "), et la société démocratique nouvellement créée doit dès lors «s'organiser pour permettre à

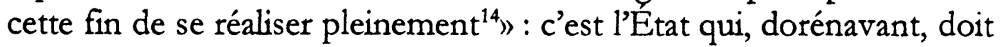
servir ses citoyens ${ }^{15}$.

D'extérieurs qu'ils étaient au départ, les obstacles à la démocratie s'avèrent, lors de ce stade ultime de développement, intrinsèques au régime récemment formé. Car la liberté nouvellement acquise «ne se conserve que par une vigilance éternelle $e^{16}$, : les institutions par lesquelles les citoyens se gouvernent doivent être sans cesse perfectionnées, adaptées aux besoins d'humains toujours changeants ${ }^{17}$. Et parce qu'en se 
transformant, les hommes transforment également leur société, il leur faut s'assurer que leur "machine gouvernementale ${ }^{18}$ " soit en mesure de faire face aux multiples modifications apportées à leur environnement : population croissante, bouleversements du territoire, bureaucratie omniprésente, industrialisation, problèmes de fédéralisme, etc. ${ }^{19}$ S'ils se laissent dépasser par l'ampleur de ces modifications, prévient Pierre Trudeau, s'ils perdent le contrôle de leur nouvelle forme de gouvernement, ils auront à composer avec un nouveau despote, ou devront apprendre à vivre dans l'anarchie la plus totale. Et Trudeau d'évoquer, à titre d'exemple, le sort de la démocratique Athènes qui, ayant négligé ses problèmes de politique extérieure, tomba aux mains de Sparte, marquant ainsi la disparition de toute démocratie sur terre pendant près de vingt siècles ${ }^{20}$.

Ce sont de telles prémisses qui vont permettre à Trudeau de brosser (en particulier au cours de la seconde moitié de la décennie) un désolant portrait du cheminement démocratique des Canadiens français. Car les obstacles à la liberté d'un peuple et les défis posés à sa démocratie sont, de l'avis de ce dernier, tributaires de la géographie et de l'histoire du pays concerné : en ce sens, s'ils veulent être en mesure de contrer efficacement les embûches placées devant eux, les citoyens d'un régime démocratique doivent tenir compte de son «évolution temporelle et spatiale ${ }^{21} \gg$ particulière. Et c'est là que le bât blesse dans le cas des Canadiens français : ceux-ci sont, selon Trudeau, incapables d'affronter les obstacles dressés devant eux, tout simplement parce qu'ils n'ont jamais assimilé le défi démocratique dans son ensemble. Ils se sont bien entendu dotés de ses éléments extérieurs, visibles (Parlement, élections, diversité des partis), mais n'ont pas encore, à ce jour, véritablement intégré la démocratie dans son essence fondamentale. Le constat est sans appel chez Pierre Trudeau : l'histoire démontre «que les Canadiens français n'ont pas vraiment cru à la démocratie pour eux-mêmes ${ }^{22}$ ). Sont ainsi cités, comme autant de preuves de cette assertion,

['] immoralisme électoral et civique des Canadiens français, [...] leur penchant pour l'autoritarisme, les thèses anti-démocratiques [sic] qu'ils apprennent au collège, les structures non-adultes où ils se débattent à l'université, le peu de place qu'ils occupent comme laïs dans l'Église québécoise, les cadres sociaux étroits où ils vivent dans les campagnes, les positions subalternes qu'ils occupent dans les structures autoritaires du capitalisme, leur crainte de recourir à l'État [et] le peu de cas qu'ils font (dans l'ensemble) des atteintes à la liberté de parole, de presse et d'association $[\ldots]^{23}$. 
Et suit la conclusion, tranchante : les exemples énumérés plus haut sont "caractéristiques d'un peuple qui n'a pas encore appris à se gouverner lui-même, d'un peuple où la démocratie ne peut pas être prise pour acquise $\left.e^{24}\right)$.

Si Trudeau dénonce, il tente également d'expliquer. Et pour ce faire, il a recours à l'argument historique : si les Canadiens français expérimentent une démocratie artificielle et incomplète, c'est qu'ils forment probablement «le seul peuple au monde qui "jouisse" du régime démocratique sans avoir eu à lutter pour l'obtenir ${ }^{25}$ ». Les "difficultés politiques particulières au Québec ${ }^{26}$ ) seraient ainsi attribuables au fait "que notre population en est arrivée au stade de la démocratie établie sans avoir traversé celui de la démocratie combattante ${ }^{27}$ \%. N'ayant jamais connu autre chose qu'un régime autoritaire avant 1763 , elle avait en quelque sorte acquis l'habitude de se soumettre aux décisions d'instances supérieures ${ }^{28}$. C'est le nouvel arrivant anglais, bien plus que le Canadien français, qui travailla à la mise en place d'un gouvernement représentatif. C'est lui qui, en 1774, réclama en vain une assemblée élective. Généralement passifs devant ces initiatives, y étant parfois même farouchement opposés, les Canadiens français n'étaient préparés, «ni psychologiquement ni politiquement ${ }^{29}$ ") à l'introduction de la Chambre représentative que marqua l'Acte constitutionnel de $1791^{30}$. Trudeau voit dans cette initiation ratée la cause première de cette conception faussée de la démocratie qu'il attribue au peuple canadien-français :

[Les Canadiens français] se trouvaient à s'initier au gouvernement responsable par le mauvais bout : un peuple auquel on n'avait jamais confié le gouvemement d'une paroisse recevait le pouvoir d'influencer par ses votes la destinée d'un État. De plus - et c'était bien naturel pour un peuple vaincu - ils apprécièrent leur nouvelle forme de gouvernement non pas tant pour ses vertus intrinsèques que comme un moyen d'assurer leur survivance ethnique et religieuse ${ }^{31}$.

Certes, concède Trudeau, les Canadiens français se lancèrent dans le nouveau processus politique, souvent même «à corps perdu ${ }^{32}$ ), mais toujours dans des buts détournés. De l'avis de ce dernier, les Rébellions des Patriotes de 1837-1838 avaient ainsi beaucoup plus à voir avec un désir féroce de survivre en tant que nation qu'avec une conversion totale au parlementarisme : celui-ci leur apparaissait tout simplement comme étant l'instrument le plus susceptible de leur permettre d'atteindre ce but ${ }^{33}$. Voilà pourquoi, renchérit-il, les Canadiens français «ne votèrent jamais pour une idéologie économique ou politique, mais 
seulement pour l'homme ou le groupe qui défendait leurs droits ethni$\left.q^{2} e^{34}\right)$. Le nationalisme fut ainsi placé au-dessus de l'idéal démocratique : le peuple canadien-français adopta la démocratie à de seules fins utilitaires, "sans adhérer profondément aux principes moraux qui [la] sous-tendent ${ }^{35} \%$. Les citoyens, pourtant distincts de leur nation, n'apprirent ainsi jamais la valeur du vote individuel, «ne considér[ant] le suffrage universel que pour sa valeur de matraque ${ }^{36} \%$.

Le constat apparaît sévère; il est heureusement tempéré. Car si Trudeau déplore le «détournement» de la démocratie qu'aurait engendré l'instinct de survie des Canadiens français, il concède tout de même l'existence de "facteurs extérieurs» aggravants à cet état de fait, des "circonstances atténuantes» en quelque sorte pour le peuple conquis. Le principal de ces facteurs? Le colonisateur anglais. Le jugement de Trudeau à ce sujet est sans équivoque, et longuement discuté dans «De quelques obstacles à la démocratie au Québec) : pour qu'une démocratie parlementaire fonctionne efficacement, commence-t-il par expliquer, il faut que les partis en présence s'entendent sur un but commun à poursuivre - le bien général - garantissant l'égalité des chances pour. tous. En ce sens, une partie des citoyens d'un pays ne peut être perpétuellement condamnée à un statut minoritaire ${ }^{37}$. Il est impératif «pour une vraie démocratie de permettre que les minorités politiques se transforment périodiquement en majorités ${ }^{38}$ \%. C'est ce postulat fondamental qui, selon la thèse trudeauiste, aurait été faussé lors de la Conquête. Car le but premier des Canadiens français ne fut jamais autre chose que d'obtenir la pleine égalité avec les Canadiens anglais, exigence qui ne fut jamais considérée sérieusement par ces derniers, ni alors ni depuis ${ }^{39}$. Mésentente sur le bien général à poursuivre donc, qui aurait empêché le groupe minoritaire d'intégrer pleinement les règles démocratiques :

En deux mots, un tiers de la nation était en désaccord avec le bien commun tel que le définissaient les deux autres tiers. Par conséquent, le gouvernement parlementaire ne pouvait fonctionner, car, dans ces circonstances, la majorité et la minorité se trouvaient foncièrement scindées l'une de l'autre et ne pouvaient altemer au pouvoir. C'est peut-être qu'après 1760, les Français furent tout aussi irréalistes dans leurs exigences que les Anglais furent intraitables dans leurs attitudes ${ }^{40}$.

Analysés sous l'angle trudeauiste, les événements subséquents de l'histoire canadienne ne sont que le résultat d'un cheminement tracé à partir de cet obstacle initial. Réclamant les avantages du gouvernement responsable pour eux-mêmes, mais refusant que ces avantages profitent également à un peuple assujetti, les Anglais visèrent d'abord, 
par la Proclamation royale, l'assimilation complète des Canadiens français. Ils attaquèrent ensuite la liberté de foi et de langue, protégée par l'Acte de Québec et rempart pourtant de toute société démocratique ${ }^{41}$. Ils s'assurèrent enfin, par l'Acte constitutionnel de 1791, d'être investis des véritables pouvoirs grâce à uun système de nomination aux Conseils exécutif et législatif ${ }^{42}$ ». La conclusion de Trudeau est catégorique; pour lui, "[l] histoire de la démocratie au Bas-Canada [...] n'est que le long récit de la perversion du systèmé lui-même ${ }^{43}$ ).

Ce n'est pas sans ironie que Trudeau fait remarquer que la situation ne commença à changer que vers 1850 , lorsque les anglophones du Bas-Canada, désormais unis à ceux du Haut-Canada, devinrent à leur tour majoritaires au pays. Ce fut comme s'ils redécouvraient soudainement le véritable sens du régime politique qu'ils avaient contribué à implanter, constate-t-il :

De ce moment, les Canadiens anglais furent à même de prêcher la grandeur de la vraie démocratie et d'appuyer leur prédication sur leur propre soumission vertueuse à la loi de la majorité. Malheureusement, il était trop tard pour faire oublier aux Canadiens français leurs premières soixante-quinze années d'enseignement démocratique, période durant laquelle l'idée de gouvemement représentatif s'était confondue avec la domination d'une minorité anglophone. En 1867, on pouvait donc difficilement s'attendre à les voir saluer comme un grand jour l'avènement d'un système de représentation fondé sur la population, puisqu'il ne pouvait maintenant s'agir que de la continuation de la domination anglophone, cette fois majoritaire ${ }^{44}$.

Si Trudeau n'atténue donc en rien ses critiques à l'égard des Canadiens français, qui ont dénaturé la démocratie en la transformant en simple arme défensive, il n'en impute cependant pas moins une partie de la faute aux Anglais. Ainsi rappelle-t-il que «si les Canadiens français firent l'erreur d'utiliser la démocratie comme un instrument de lutte ethnique, les Canadiens anglais leur offrirent d'amples occasions de la concevoir ainsi ${ }^{45}$ \%. Les francophones ont sans l'ombre d'un doute commis une erreur en percevant la démocratie comme une institution étrangère, éloignée de leur histoire particulière ${ }^{46}$ et de leurs caractéristiques fondamentales; jamais cependant le peuple conquérant ne leur a fourni l'occasion de voir les choses autrement. Le jugement qui conclut l'argumentation est empreint de sévérité : «[...] les Canadiens anglais se sont pendant longtemps comportés, en politique nationale, comme s'ils croyaient que la démocratie n'était pas faite pour les Canadiens français ${ }^{47}$ ). 
S'il affirme que l'hypocrisie canadienne-anglaise constitue l'élément majeur des obstacles «extérieurs» ayant freiné le cheminement démocratique du Canada français, Trudeau refuse cependant de faire peser la faute sur les seules épaules du conquérant anglophone. Au sein de la nation canadienne-française même, prétend-il, des embûches «officielles» se sont dressées afin de contrer une assimilation totale, par les citoyens francophones, des principes et visées du système parlementaire. L'Église catholique, par exemple, s'est efforcée de maintenir, dans un régime censément démocratique, tous les attributs du totalitarisme. Les reproches adressés à l'institution ecclésiastique sont virulents :

Peuple conquis, non seulement les Canadiens français devaient-ils faire face à un État redouté parce qu'il était la créature d'une nation étrangère, mais encore appartenaient-ils à une Église qui se méfiait de cet État, puissance rivale, enfant de la Révolution, siège possible de la domination des anti-cléricaux [sic], des protestants ou même des socialistes ${ }^{48}$.

En catholique libéral façon Esprit, Trudeau aborde les relations entre catholicisme et démocratie sous un angle critique, qui laisse peu de place à l'indulgence. Historiquement, explique-t-il, «[les] nations catholiques n'ont pas toujours été très favorables à la démocratie ${ }^{49}$ ). Autoritaires sur le plan spirituel, les catholiques, parce qu'ils ont souvent refusé de faire la distinction nécessaire entre le spirituel et le temporel, ont transposé cette autorité dans le domaine politique. Dès lors, et au Québec en particulier, le vote libre, décidé par la seule conscience individuelle, est devenu moins important que les directives électorales données en chaire ${ }^{50}$; le prêtre a eu préséance sur le citoyen, et la démocratie n'en a été que davantage faussée.

L'Église se serait donc méfiée de la démocratie parce que celleci contrevenait à sa vision d'une société idéale où le politique devait être à la solde du spirituel; elle s'en serait également méfiée, seconde conclusion de l'analyse de Pierre Trudeau, parce qu'elle y voyait une menace à son rôle de protectrice de la foi. La Conquête, quoi qu'on puisse en penser, l'avait en effet placée, estime-t-il, dans une position fort avantageuse : elle demeurait le seul guide des Canadiens français, et pouvait éventuellement représenter pour les Canadiens anglais un adversaire redoutable. Un accord de (nnon-agression) entre les deux partis semblait, dans ces circonstances, aller de soi. Les catholiques seraient libres de pratiquer leur religion; en échange, leur Église se montrerait loyale à la Couronne anglaise. Et Trudeau d'évoquer, comme preuves de cette alliance officieuse, la soumission prêchée par les autorités ecclésiastiques lors des guerres de 1914 et de 1939, l'appel lancé contre les Féniens en 
1870 , le désaveu du mouvement patriote, etc..$^{51}$ De l'avis du polémiste, la constatation s'impose : «[l]a foi étant assurée, les hommes d'Église ne se soucièrent guère [par la suite] de la liberté démocratique ${ }^{52}$ ).

La boucle est ainsi bouclée; le constat chez Trudeau apparaît limpide. Dans leurs relations avec l'État, les Canadiens français sont 《immoraux ${ }^{53}$ »: ils abusent du système, musellent leurs adversaires trop bruyants, recherchent leur seul profit. Ils ont, au fil des ans, "form[é] une communauté dans la communauté», cherchant à préserver la première en abusant des règles établies par la seconde ${ }^{54}$. Parce qu'ils n'ont jamais eu à se battre pour obtenir la démocratie, qu'ils ne sont pas en ce sens à l'origine du régime, les Canadiens français l'ont toujours perçu comme un phénomène étranger. Ils en ont bénéficié, et en ont usé, mais toujours comme d'une stratégie $e^{55}$. Plutôt que de voir l'État comme un produit de leur société, chargé d'assurer leur mieux-être collectif, ils en sont venus à le percevoir comme un agent à la solde de l'ennemi anglophone, colonisateur, athée ${ }^{56}$... Encouragés en ce sens tantôt par le conquérant anglais, tantôt par l'Église catholique, ils n'ont jamais été en mesure de participer pleinement à son développement ${ }^{57}$. De là, selon Trudeau, l'incivisme d'un peuple qui n'a jamais expérimenté une véritable démocratie :

[...] au Québec, où chacun sait que l'autorité vient de Dieu, on n'attache guère d'importance aux simagrées électorales : ce sont des divertissements protestants et anglo-saxons dont la signification profonde reste obscure, et dont l'utilité immédiate se traduit par la bouteille de whisky à recevoir, par la salle paroissiale à faire bâtir, ou par le contrat de route à obtenir ${ }^{58}$.

Si les critiques de Trudeau sont abondantes et explicites, les solutions proposées le sont de leur côté beaucoup moins. Elles font appel aux grands principes, à certains thèmes fétiches chez le penseur : le rationalisme, la liberté, le fonctionnalisme. L'objectif ultime demeure la concrétisation d'une véritable démocratie au Canada français. Les exhortations en ce sens se font pressantes au cours des années 1950 : Trudeau rappelle en effet à maintes reprises que «la dialectique de l'action nous impose impérieusement [aux Canadiens français] de concentrer nos effectifs sur un objectif unique : la démocratie ${ }^{59} \%$. Le peuple doit «commencer à apprendre la démocratie en partant de zéro ${ }^{60} \%$. Il lui faut ainsi «fabriquer l'enveloppe démocratique avant de se diviser sur la définition de son contenu ${ }^{61} \%$. Les moyens évoqués pour atteindre cette fin tiennent parfois plus du lyrisme littéraire que de la pure analyse po- 
litique. Pour Trudeau, il faut en effet uretrouver l'audace des évangélisateurs, des explorateurs, des coureurs des bois, et être prêts comme eux $\grave{a}$ risquer le tout pour le tout ${ }^{6}{ }^{2}$ \%. À cet effet, il importe d'être "chrétiens au point d'aimer tous les hommes comme nos frères», afin d'éviter l'existence «dans la nation $\mathrm{d}[\mathrm{e}]$ groupes compartimentés suivant leurs intérêts ${ }^{63}[\ldots] \gg$ On en revient finalement à cette nécessité d'éliminer de la «nouvelle» démocratie tout aspect utilitaire ou intéressé. Dans un article où il traite plus spécifiquement de ce sujet, Pierre Trudeau dresse le programme à suivre pour favoriser chez les Canadiens français un véritable sens de l'État:

[...] il faudra s'en prendre à toutes les autres panacées politiques, et ne rien accepter qui ne puisse se justifier en termes positifs et raisonnables. J'entrevois une étude approfondie de notre système fédéraliste, un examen sévère de nos postulats bilinguistes [sic], une séparation définitive de l'Église et de l'État, une psychanalyse de notre infantilisme religieux et culturel, une mise en accusation de nos soi-disant élites, et bien d'autres enquêtes, dans l'espoir de rassembler les éléments d'une politique vraiment humaine ${ }^{64}$.

Sans doute ne le prévoyait-il pas au moment où il écrivait ces lignes (en 1950), mais cette critique de l'infantilisme religieux et culturel au Canada français, cette mise en accusation des élites et toutes ces "autres enquêtes» allaient devenir réalité quelques années plus tard, résultats d'une importante recherche dont Trudeau serait l'un des maitres d'œuvre. Publiée sous le titre La grève de l'amiantes , la charge virulente à l'endroit des institutions traditionnelles canadiennes-françaises qui résulterait de ces travaux n'allait pas manquer de provoquer les réactions les plus diverses, de l'enthousiasme le plus débordant à la dénonciation la plus violente. De doléances somme toute encore générales, Trudeau passerait alors aux accusations directes : c'est tout un pan de la société canadienne-française qui serait sévèrement attaqué, marquant ainsi la concrétisation d'un cheminement idéologique amorcé quelques années auparavant.

\section{NOTES}

'Pierre Elliott Trudeau, "Avant-propos », Le fédéralisme et la sociêté canadienne-francaise, Montréal, Éditions HMH Ltée., 1967, p. IX.

${ }^{2}$ Ibid.

${ }^{3}$ Gérard Pelletier, «Introduction», dans Idem, Réponses de Pierre Elliott Trudeau, Montréal, Les Éditions du Jour, 1968, p. 22.

"Pierre Elliott Trudeau, «Politique fonctionnelle», Cité libre, n 1 (juin 1950), p. 21.

${ }^{5}$ Ibid. 
"Idem, "Obstacles à la démocratie», Le Peuple souverain. Rapport de la Première Conférence Annuelle de l'Institut canadien des affaires publiques, [s. 1.], 1954, pp. 36-40.

${ }^{7}$ Voir en particulier Idem, "Politique fonctionnelle», Cité libre, n 1 (juin 1950), pp. 20 24 et «Réflexions sur la politique au Canada français», Cité libre, n 3 (décembre 1952), pp. $53-70$.

${ }^{8}$ Les conférences de l'Institut canadien des affaires publiques sont diffusées sur les ondes de la Société Radio-Canada.

${ }^{9}$ Nous empruntons la terminologie de Pierre Trudeau.

10 Trudeau, "Obstacles à la démocratie», loc. cit., pp. 36-37.

11 Ibid., p. 36.

12 Ibid., p. 37.

${ }^{13}$ Ibid.

${ }^{14}$ Ibid.

${ }^{15}$ Ibich

${ }^{16} \mathrm{Idem}$, «Un manifeste démocratique», Cité libre, n ${ }^{\circ} 22$ (octobre 1958), p. 17.

${ }^{17}$ Idem, "Obstacles à la démocratie», loc. cit., p. 37.

${ }^{18}$ Ibid.

${ }^{19}$ Idem, «Un manifeste démocratique», loc. cit., p. 17.

20 Idem, "Obstacles à la démocratie», loc. cit., p. 37.

${ }^{21}$ Ibid., p. 38.

22 Idem, "De quelques obstacles à la démocratie au Québec», dans Le fédéralisme et la societé canadienne-franfaise, Montréal, Éditions HMH Ltée,, 1967, p. 107. L'article retenu ici a été publié pour la première fois plusieurs années auparavant dans le Canadion Journal of Economics and Political Science, Toronto, vol. XXIV, n 3 (août 1958), pp. 297 311.

${ }^{23}$ Idem, "Un manifeste démocratique», loc. cit., pp. 17-18.

${ }^{24}$ Ibid., p. 18.

${ }^{25} \mathrm{Idem}$, «De quelques obstacles...", loc. cit., p. 107.

${ }^{26}$ Idem, "Obstacles à la démocratie», loc. cit., p. 38.

${ }^{27}$ Ibid.

${ }^{28}$ Idem, " De quelques obstacles... ", loc. cit., p. 108.

${ }^{29}$ Ibid.

${ }^{30}$ Ibid.

${ }^{31}$ Ibid. (Nous soulignons.)

${ }^{32}$ Idem, "Réflexions sur la politique...», loc. cit., p. 54.

${ }^{33}$ Trudeau rajoutera même, à propos des Patriotes : «[...] leur secret dessein, en adoptant morceau par morceau le système britannique, ce n'était pas seulement d'utiliser celui-ci, mais d'en abuser s'il le fallait.» Voir «De quelques obstacles...», loc. cit., p. 109.

${ }^{34}$ Ibid., p. 111.

${ }^{35}$ Ibid., p. 110.

${ }^{36}$ Idem, "Obstacles à la démocratie», loc. cit., p. 39.

${ }^{37}$ Idem, «De quelques obstacles", loc. cit., pp. 118-119.

${ }^{38}$ Ibid., p. 119.

${ }^{39}$ En 1958.

${ }^{40}$ Idem., "De quelques obstacles.... ", loc. cit., p. 119. 
"1 Ibid., pp. 119-120.

42 Ibid., p. 120.

${ }^{43}$ Ibid.

4t Ibid., pp. 121-122. 'Trudeau mentionne de plus certains événements qui, à son avis, contribuèrent par la suite à confirmer cette impression initiale défavorable chez les francophones : l'élimination du bilinguisme à la législature du Manitoba, la formation d'un gouvernement d'union en 1917, l'emploi du plébiscite en 1942, la pratique visant à favoriser l'immigration britannique aux dépens de l'immigration française, etc.

${ }^{45}$ Ibid., p. 122.

${ }^{46}$ L'argumentation de Pierre Trudeau n'est pas sans contradiction. Ainsi, s'il mentionne ici que la vision des Canadiens français, qui perçurent longtemps la démocratie comme étant une institution étrangère à leur histoire particulière, fut une «erreun de la part de ces derniers, lui-même a pourtant, plus haut, analysé l'esprit «antidémocratique» canadien-français sous cet angle. Voir à cet effet les notes 25 à 31 du présent article.

${ }^{47}$ Ibid., p. 124.

${ }^{48}$ Ibid., p. 113.

49 Ibid, p. 112.

${ }^{50}$ Ibid. Pierre Trudeau exprimait déjà cette idée en 1952, alors qu'il écrivait dans «Réflexions sur la politique au Canada français»: "[...] il faut reconnaittre que les catholiques, en tant que collectivité, ont rarement été des piliers de la démocratie - je le dis à notre grande honte. Car ils en arrivent à si bien mêler les questions spirituelles et temporelles, qu'ils se résignent difficilement ensuite à statuer d'aucune vérité en comptant des votes. Et dans les pays à grande majorité catholique (Pologne, Autriche, Espagne, Portugal, Italie, Républiques Sud-Américaines [sid] [...]) souvent ils n'échappent à l'anarchie que par l'autoritarisme, tandis que là où leur force est moindre (France, Allemagne, Pays-Bas, Belgique, États-Unis, Canada) ils acceptent la séparation de l'Église et de l'État comme un pis-aller, ils consacrent leurs énergies civiques à la poursuite du bien particulier catholique et, comme groupe, ils ne se signalent ni par leur moralité ni par leur clairvoyance politiques.» (pp. 55-56.)

st Ibid., p. 112.

${ }^{52}$ Ibid.

${ }^{53}$ Idem, «Réflexions sur la politique...,", loc. cit., p. 53.

${ }^{54}$ Ibid., p. 55.

55 «Une casuistique subtile nous avait autorisés à enfreindre certaines règles du jeu politique, et voilà qu'à la fin le jeu tout entier glissa hors du champ de la moralité. Nous avions si bien subordonné le bien commun (canadien) au bien particulier (canadienfrançais) que nous perdîmes le sens moral de notre obligation vis-à-vis du premier. Et hors des temps de crise (Riel, écoles séparées de l'Ouest, conscription), où nous nous ralliions d'instinct pour assurer le bien particulier de notre survivance au sein de la communauté globale, chacun se crut en droit de poursuivre, au détriment de celle-ci, son bien particulier à lui. C'est dire que notre sens civique fut perverti; nous devinmes des opportunistes.» Voir Ibid.

${ }^{56}$ Idem, "Saper la majesté de l'Étað, Les cheminements de la politique, Montréal, Éditions du Jour, 1970, pp. 53-54.

${ }^{57}$ L'analyse que fait ici Pierre Trudeau laisse entrevoir des limites évidentes. Ainsi, l'opportunisme politique apparaît être, sous sa plume, une réalité propre au Canada français pré-Révolution tranquille. Trudeau semble oublier qu'une fraction du corps 
électoral, peu importe les pays, est toujours opportuniste, et fait souvent pencher la balance de son côté. Sa vision des choses, parce qu'elle oublie de faire les distinctions nécessaires entre les époques, est également anachronique : Pierre Trudeau parle de démocratie en 1790 comme en 1837 ou en 1950 . Il ne distingue pas le parlementarisme libéral, longtemps prérogative de quelques privilégiés seulement, de la véritable démocratie, dont les prétentions sont universelles. La Grande-Bretagne des XVIII et $\mathrm{XIX}^{\mathrm{e}}$ siècles demeure par exemple, malgré son régime parlementaire, le bastion d'une démocratie encore toute relative, d'où la plupart sont exclus. La corruption, l'opportunisme et l'égoïsme des intérêts particuliers, loin d'être des phénomènes uniques au Canada français, ont, de tout temps, fait partie intégrante des systèmes politiques américain, britannique et français, entre autres. La «démocratie» de Trudeau est un idéal, non une réalité politique.

${ }^{58} \mathrm{Idem}$, «Réflexions sur la politique...», loc. cit., p. 56.

${ }^{59}$ Idem, «Un manifeste démocratique», loc. cit., pp. 19-20.

${ }^{\circ 0} \mathrm{Idem}$, «De quelques obstacles...», loc. cit., p. 118.

${ }^{\circ 1}$ Idem, «Un manifeste démocratique», loc. cit., p. 20.

${ }^{02}$ Idem, "Obstacles à la démocratie», loc. cit., p. 40.

${ }^{63} \mathrm{Idem}$, "Réflexions sur la politique», loc. cit., p. 70.

${ }^{64}$ Idem, "Politique fonctionnelle», loc. cit., pp. 23-24. Il est intéressant de constater à quel point la perception de Pierre Trudeau au sujet de «l'immoralisme» démocratique des Canadiens français sera modifiée à la suite de la Révolution tranquille. C'est en effet un Trudeau nettement plus indulgent à l'endroit de ses compatriotes qui écrira en 1968: "Les choses que je disais ont cessé d'être vraies à partir du moment où les Canadiens français ont appris la démocratie pour eux-mêmes et ont commencé à s'en servir [...], et à partir du moment où les Canadiens anglais se sont rendu compte que la démocratie ne devait pas seulement servir la majorité numérique du pays mais l'ensemble des citoyens [...]. Les situations que je décrivais il y a 10 ans ont donc été solutionnées. Et c'est ce qui me fait penser que les Canadiens français sont maintenant assez mûrs politiquement pour pouvoir se gouverner provincialement [sic] et pour pouvoir participer, à part entière, à un gouvernement fédéral. Voir à cet effet Idem, Réponses de Pierre Elliott Trudeau, Montréal, Les Éditions du Jour, 1968, p. 50.

${ }^{65}$ Idem, dir., La grève de l'amiante, Montréal, Les Éditions du Jour, 1970 [1956], 430 pages. 